ene

\title{
Plan de cuidados para ancianos CON DEPRESIÓN: A PROPÓSITO DE UN CASO
}

\section{Care plan for Depression in the elderly. ABOUT A CASE}

Rosana Gómez Conte, Cristina Cases Jordán

Centro Residencial Ancianos Madre de Dios de Begoña 


\section{Resumen}

La depresión es una enfermedad mental que cursa entre otros síntomas con alteración del estado de ánimo. Existen multitud de tratamientos en los que se encuadran psicofármacos además de otras terapias entre las que destaca la movilidad física. Se ha demostrado que el ejercicio tiene grandes beneficios que mejoran claramente los síntomas de depresión geriátrica. Cabe destacar que enfermería tiene un papel indispensable en la detección precoz de los síntomas, la realización de diagnósticos enfermeros y la ejecución correcta de las actividades de enfermería.

Se realizó un plan de cuidados a un paciente anciano con depresión de una Residencia de Zaragoza. Las intervenciones estaban relacionadas con el aumento de la movilidad física para reducir la depresión en esta persona.

Se realizó una búsqueda bibliográfica de artículos científicos a través de diversas bases de datos y se consultaron libros disponibles en la biblioteca de la Universidad de Zaragoza. Para la realización del plan de cuidados se utilizó el marco de valoración de Marjory Gordon y sus 11 patrones funcionales de salud y las etiquetas diagnósticas de la taxonomía NANDA-I así como los criterios de resultados (NOC) y las intervenciones (NIC).
La movilidad física ayuda a mejorar la depresión en el paciente geriátrico realizando un abordaje global. Esto se llevará a cabo mediante la confianza del paciente en el profesional de enfermería, la expresión de sentimientos y el apoyo junto con el aumento de la movilidad física.

Palabras Clave: Ancianos, Depresión, Enfermería, Ejercicio, Factores de riesgo. 


\section{Abstract}

Depression is a mental illness which deals with alteration of mood, among other symptoms. There is a high variety of treatments like psychotropic drugs. There are also other therapies, among which physical mobility stands out. It has been tested that exercise has intense benefits which clearly improve the symptoms of geriatric depression. It should be noticed that nursing has an essential role in the early detection of symptoms, diagnostics development and proper execution of nursing activities.

A care plan for an elderly patient with depression was carried out in a residence in Zaragoza. Activities were related to physical mobility increase in order to reduce depression in this person.

A bibliographical search of scientific papers has been made through several databases. Some available books in the library of the University of Zaragoza were consulted as well. Marjory Gordon's model and its 11 functional health patterns, NANDA taxonomy Diagnostics Labels, and Results Criteria (NOC) and interventions (NIC) were used for the care plan

Physical mobility helps to improve depression in the geriatric patient, doing a global approach. This project will be carried out through the patient confidence in the professional, the feelings ex- pression and the support all together with the physical mobility increase.

Key words: elderly, depression, nursing, exercise and risk factors. 


\section{INTRODUCCIÓN}

La depresión se encuadra dentro del marco de los trastornos afectivos (del humor) cursando con síntomas muy amplios. Dichos síntomas se encuadran desde lo puramente sentimental - tristeza, pesimismo, ánimo bajo - hasta síntomas somáticos, dando anomalías en la entrevista diagnóstica. (1)

Es evidente que no pueda hablarse de un único agente etiológico de la depresión. Es preciso tener en cuenta la vulnerabilidad psicológica de la persona, así como circunstancias que bloqueen la capacidad de enfrentamiento: (2) se incluye el estrés, el tabaquismo y el consumo de alcohol, el desempleo y una alimentación poco saludable, además de enfermedades crónicas. (3)

El gran problema diagnóstico de la depresión y de las enfermedades mentales es la no existencia de un síntoma definitorio que marque el diagnóstico de certeza deseado. (1) Esta es la razón por la que aparece el llamado signo "Fenómeno de iceberg", en el que un gran número de depresiones nunca llegan a ser tratadas. (2)

Además, comparando con otras enfermedades crónicas como la cardiopatía o la diabetes, cuanto más temprana sea la intervención, mejor será la evolución. Por ello el tratamiento debe ser lo más precoz posible. (4)
Es de especial interés la detección del riesgo de suicidio como diagnóstico enfermero. La prevalencia ronda el $20 \%$ en Estados Unidos siendo los ancianos el grupo etario con mayor riesgo de dicho diagnóstico. (5) La prevención va a resultar crucial a través de actividades individuales y poblacionales. (1)

El estudio del Epidemiologic Catchment Area indica una prevalencia de depresión del 5,8 \% en la población americana hasta los 84 años de edad. (1) Otros estudios como el Estudio Zaragoza que se realizó con una muestra de 1.500 enfermos señalan que la prevalencia es del $7 \% .(6,7)$

Los datos apuntan que el número de adultos mayores está creciendo significativamente. La Organización Mundial de las Naciones Unidas prevé que para el año 2.025, 1.100 millones de personas serán ancianos. (8) Todo esto trae consigo la progresiva dependencia para las actividades de la vida diaria. (9) La frecuencia de depresión en mayores fue del $72,1 \%$ en un estudio realizado en del Estado de México. Así pues, se entiende a la población anciana como el mayor grupo vulnerable a esta enfermedad. (10)

Igual que en la población general, los factores relacionados de la depresión en ancianos también varían. Desde variables demográficas como edad o sexo, hasta condiciones inadecuadas de salud, 
como enfermedades crónicas (trastornos respiratorios, artritis y alteraciones vasculares), (12) además de la necesidad de vivir institucionalizados. También puede influir en la aparición de la depresión una inadecuada red familiar. $(12,13)$ Por tanto, se considera la depresión en ancianos como un problema de salud pública. (14)

Los DSM-IV son unos criterios internacionales que ayudan en el reconocimiento de la depresión (Anexo 1). Además existen escalas que valoran la salud mental en los ancianos como la escala de depresión de Hamilton y la escala de Depresión Geriátrica Yesavage, entre otras. $(15,8)$

El tratamiento de la depresión en ancianos engloba también la asistencia multidisciplinar a través de programas de intervención óptimos. Un ejemplo es la actividad física moderada. La práctica del ejercicio físico se relaciona con el bienestar y la vida saludable. (15) Así pues, se pueden clasificar los beneficios de la actividad física en fisiológicos (mejora de los sistemas cardiovascular, respiratorio, músculo-esquelético, locomotor) y psicológicos. ${ }^{(11)}$

Muchos estudios han determinado las ventajas del ejercicio físico en los mayores. En un estudio con 57 ancianos institucionalizados del municipio de Villaviciosa de Odón (Madrid) se demostró que aquellos más sedentarios padecían más depresión que los que realizaban actividad física moderada o en grupo. (16)

Por lo tanto, durante el envejecimiento, la movilidad física representa un buen tratamiento adyuvante para problemas físicos y desórdenes mentales como la depresión y la ansiedad. $(16,17)$

Los beneficios de la actividad física en mayores obtenidos en diversos estudios, entre ellos uno realizado en Ciudad de La Habana, fueron:

- Disminuye los problemas osteomioarticulares al disminuir el dolor y la tensión muscular. (9)

- Mejora la higiene del sueño combatiendo el insomnio. (9)

- Mejora la calidad de vida fortaleciendo el cuerpo en general. Aumenta la seguridad ante las caídas y mejora los trastornos del equilibrio. Además aumenta la fuerza y la habilidad para realizar actividades. $(9,18,19)$

- Mejora la circulación y los niveles de colesterol evitando así cualquier evento coronario. (8)

- Evita el estreñimiento, y mejora el Índice de Masa Corporal. (8)

A nivel psicológico disminuye el estrés, facilita la relajación y mejora la autoestima y la función cognitiva. (8) 
- Mejora el estado de ánimo

y ofrece distracción. Con la actividad física se evita el aislamiento social fomentando las actividades en grupo. (9)

- Mejora la depresión a corto plazo por su efecto antidepresivo y ansiolítico gracias a sus propiedades antiinflamatorias.

No obstante, se debería tener especial atención en mayores con cardiopatías y problemas cardiovasculares ya que algunos de estos podrían ser contraindicaciones absolutas como la insuficiencia cardiaca aguda. (21)

Se recomienda considerar los psicofármacos y la psicoterapia en el tratamiento de la depresión en ancianos. (22) Además diversas terapias como la electro-convulsiva, adecuados hábitos dietéticos y la movilidad física mejoran claramente los síntomas de depresión geriátrica. $(23,24)$ Las actividades físicas más recomendadas en el adulto mayor son caminar (150 minutos de actividad moderada a la semana, o 75 minutos de actividad intensa a la semana), ejercicios gimnásticos, natación e ir en bicicleta. (25)

Se entiende que el abordaje de enfermería es indispensable para brindar una atención de calidad al adulto mayor. (26) La identificación de los problemas de salud y el planteamiento de las actividades de enfermería se hace indispensable. La cercanía que enfermería tiene con los pacientes posibilitará una mejor valoración a través de la detección precoz de los síntomas, así como la realización adecuada de los diagnósticos correspondientes. $(27,28)$

\section{OBJetivo}

Realizar un plan de cuidados a un paciente anciano con depresión de una Residencia de Zaragoza.

Establecer intervenciones que aumenten la actividad física de dicho paciente para reducir la depresión en esta persona a través del aumento de la movilidad.

En relación a los criterios éticos se proporcionó un consentimiento informado revisado por la Universidad de Zaragoza a la paciente que aceptó participar en el plan de cuidados. Así mismo, la enfermera firmó un documento de protección de datos de acuerdo a la Ley Orgánica $3 / 2018$, de 5 de diciembre, de Protección de Datos Personales y garantía de los derechos digitales.

\section{MÉTODO}

Se ha realizado una búsqueda bibliográfica durante el mes de febrero de 2016 de artículos científicos a través 
PLAN DE CUIDADOS PARA PACIENTES CON DEPRESIÓN: A PROPÓSITO DE UN CASO.

de diversas bases de datos. La finalidad

ha sido recoger información relevante acerca de la depresión en mayores y su relación con la movilidad física (tabla 1).
Se han utilizado los artículos en los que el resumen y los objetivos se adecuaban al tema que se deseaba tratar.

\begin{tabular}{|c|c|c|c|c|}
\hline BASE DE DATOS & $\begin{array}{l}\text { DESCRIPTORES/ } \\
\text { PALABRAS CLAVE }\end{array}$ & FILTROS ACTIVADOS & $\begin{array}{l}\text { ARTÍCULOS } \\
\text { REVISADOS }\end{array}$ & $\begin{array}{l}\text { ARTÍCULOS } \\
\text { UTILIZADOS }\end{array}$ \\
\hline Pubmed & $\begin{array}{c}\text { Aged } \\
\text { Exercise } \\
\text { Depression }\end{array}$ & $\begin{array}{c}\text { Abstract, Free full text, } \\
\text { published in the last } 5 \\
\text { years, Humans, English, } \\
\text { Spanish. }\end{array}$ & 22 & 9 \\
\hline Cuiden & $\begin{array}{l}\text { Ancianos } \\
\text { Depresión } \\
\text { Enfermería }\end{array}$ & $\begin{array}{l}5 \text { últimos años, texto } \\
\text { completo, original. }\end{array}$ & 4 & 4 \\
\hline Scielo & $\begin{array}{l}\text { Ancianos } \\
\text { Depresión } \\
\text { Ejercicio }\end{array}$ & Todos los índices & 4 & 3 \\
\hline Science direct & $\begin{array}{c}\text { Depresión } \\
\text { Adulto mayor } \\
\text { Frecuencia } \\
\text { Factores de riesgo }\end{array}$ & Atención Primaria & 2 & 2 \\
\hline Libre elección & & & 6 & 6 \\
\hline $\begin{array}{c}\text { Libros de la biblioteca } \\
\text { de la universidad de } \\
\text { Zaragoza }\end{array}$ & & & 4 & 4 \\
\hline
\end{tabular}

Tabla 1. Información relevante acerca de la depresión en mayores y su relación con la movilidad física

Para la realización del plan de cuidados de esta paciente se ha utilizado el método de valoración de Marjory Gordon y sus 11 patrones funcionales de salud.

Además se ha realizado la escala de depresión de Yesavage y el mini examen cognitivo de Lobo para cuantificar la depresión y el estado cognitivo de la paciente.
Las Etiquetas Diagnósticas de la taxonomía NANDA-I así como los Criterios de Resultados (NOC) y las Intervenciones (NIC) han sido utilizados para establecer el plan de atención de Enfermería.

\section{Plan de Cuidados}

\section{VALORACIÓN}

Mujer mayor de 65 años, viuda, autónoma. 
Sus antecedentes personales son cólico biliar y hernia de hiato. La paciente refiere dolor leve que se alivia con tratamiento farmacológico.

Tras un ingreso hospitalario por patología digestiva aguda que coincide en el tiempo con el fallecimiento de su único hijo ingresa en una residencia de Zaragoza aconsejada por los servicios sociales.

Era una señora activa, con buena movilidad física, pero su estado anímico cambió drásticamente por el fallecimiento de su hijo y con el agravante de no poder acompañarlo en sus últimos momentos. Así pues, perdió su único apoyo familiar, su casa y la motivación para realizar las actividades básicas de la vida diaria en un corto periodo de tiempo.

En pleno proceso de duelo está luchando por seguir realizando sus actividades habituales anteriores pero no dispone de la fuerza y el apoyo necesario en la actualidad.

Sale a la calle sola pero no de forma prolongada, ya que no tiene la confianza para ello.

Le gusta relacionarse con la gente y expresa esta necesidad de manera constante. Se muestra colaboradora y contenta por el plan a realizar.

Realizada escala de Depresión Yesavage cuyo resultado diagnostica Depresión Leve con una puntuación de nueve (29) (Anexo 2); y Mini Examen Cognoscitivo de Lobo con deterioro cognitivo leve en el que obtiene una puntuación total de veinticuatro. (30)

Se ha valorado cada uno de los patrones funcionales de Marjory Gordon para la paciente, localizando una alteración en los siguientes:

Uno: Percepción-Manejo de la Salud. La paciente tiene conciencia de sufrir una alteración del estado de ánimo, aunque ella niega padecer depresión.

Además presenta sentimiento de minusvalía personal, social y preocupación excesiva sobre lo que le rodea.

Realizada la escala de Yesavage para valorar el estado de ánimo de la paciente.

Dos: Nutricional-Metabólico. La depresión le ha producido a la paciente anorexia con la consiguiente pérdida de peso.

Cuatro: Actividad-ejercicio. La pasividad, la inhibición psicomotriz y la falta permanente de energía para realizar cualquier actividad han aumentado en la paciente. Además, han disminuido las ganas para realizar las actividades de autocuidado y las domésticas. 
Seis: Cognitivo-Perceptivo. La paciente padece alteraciones en la percepción del entorno así como dificultades para concentrarse y de memoria.

Realizado el Mini Examen Cognoscitivo de Lobo para valorar el deterioro cognitivo.

Siete: Percepción de sí mismoauto concepto. La paciente siente una percepción pesimista y negativa de sí misma y vacío vital a su alrededor.

Diez: Afrontamiento-tolerancia al estrés. La paciente tiene sentimientos de impotencia, inutilidad y frustración.

\section{DIAGNÓSTICO}

A partir de los patrones de $\mathrm{M}$. Gordon que se encuentran alterados en la etapa de valoración, se define este diagnóstico de enfermería según la taxonomía NANDA-I. (31)
00137 Aflicción crónica r/c muerte de un ser querido $m / p$ expresión de uno o varios de los sentimientos negativos siguientes: depresión, vacío, frustración, desesperanza, soledad, baja autoestima.

Dominio 9: Afrontamiento/tolerancia al estrés.

Clase 2: Respuestas de afrontamiento.

Definición: Patrón cíclico, recurrente y potencialmente progresivo de tristeza generalizada experimentado en respuesta a una pérdida continua, en el curso de una enfermedad o discapacidad.

En este caso viene dada por la pérdida de su hijo, de su casa y el deterioro progresivo de su salud.

\section{PLANIFICACIÓN}

Dado el diagnóstico de enfermería establecido para la paciente, se han elaborado objetivos e intervenciones relacionados a través del lenguaje NIC Y NOC. $(32,33)$

\begin{tabular}{|c|c|c|c|}
\hline CLASE & INTERVENCIÓN & RESULTADOS & INDICADORES \\
\hline $\begin{array}{l}\text { Control de la actividad y } \\
\text { ejercicio. }\end{array}$ & $\begin{array}{l}0200 \text { Fomento del } \\
\text { ejercicio. }\end{array}$ & $\begin{array}{l}\text { C: } 0200 \text { Ambular. } \\
\text { C: } 0208 \text { Movilidad. }\end{array}$ & $\begin{array}{l}020002 \text { Camina con marcha eficaz. } \\
020011 \text { Camina distancias moderadas. } \\
020810 \text { Marcha. } \\
020806 \text { Ambulación. }\end{array}$ \\
\hline
\end{tabular}




\begin{tabular}{|l|l|l|l|}
\hline $\begin{array}{l}\text { Control de la actividad y } \\
\text { ejercicio. }\end{array}$ & $\begin{array}{l}\text { 0221 Terapia de } \\
\text { ejercicios: ambulación. }\end{array}$ & $\begin{array}{l}\text { T: } 1909 \text { Conducta de } \\
\text { prevención de } \\
\text { caídas. }\end{array}$ & $\begin{array}{l}190910 \text { Uso de zapatos con cordones bien } \\
\text { ajustados. } \\
190915 \text { Uso de barandillas para agarrarse. }\end{array}$ \\
\hline $\begin{array}{l}\text { Potenciación de la } \\
\text { comunicación. }\end{array}$ & 4920 Escucha activa. & $\begin{array}{l}\text { J: } 0902 \\
\text { Comunicación. } \\
\text { M: } 1206 \text { Deseo de } \\
\text { vivir. }\end{array}$ & $\begin{array}{l}\text { 090202 Utiliza el lenguaje hablado. } \\
00202 \text { Dirige el mensaje de forma apropiada. } \\
120605 \text { Expresión de sentimientos. } \\
120609 \text { Utiliza estrategias para mejorar la salud. }\end{array}$ \\
\hline $\begin{array}{l}\text { Ayuda para hacer frente a } \\
\text { situaciones dif́ciles. }\end{array}$ & $\begin{array}{l}5270 \text { Apoyo } \\
\text { emocional. } \\
5290 \text { Facilitar el duelo. }\end{array}$ & $\begin{array}{l}\text { N: } 1304 \text { Resolución } \\
\text { O: } 1409 \text { Autocontrol } \\
\text { de la depresión. }\end{array}$ & $\begin{array}{l}130403 \text { Expresa sentimientos sobre la pérdida. } \\
130403 \text { Verbaliza la realidad de la pérdida. } \\
140913 \text { Sigue un programa de ejercicio. }\end{array}$ \\
\hline
\end{tabular}

Existen otras intervenciones y resultados posibles para realizar en este plan de cuidados pero no han sido trabajados debido a su menor importancia.

\section{EJECUCIÓN}

Las intervenciones especificadas en la planificación se han ejecutado a través de una serie de actividades.

Durante un mes la enfermera ha acudido una vez a la semana para realizar sus intervenciones y el resto de la semana la paciente ha realizado las actividades de manera independiente.

Además de las actividades relacionadas con la movilidad física, se han incluido una serie de acciones encaminadas a la expresión de sentimientos y a que la paciente se sintiera escuchada y acompañada.

\section{Actividades enfermera-pacien-} te: $1^{\mathrm{a}}$ intervención: (22 de Marzo de 2016)

Se establece la primera toma de contacto, una presentación mutua y el inicio de una relación de confianza. La paciente le enseña su habitación y sus pertenencias. Se muestra impaciente por comenzar el plan de movilidad física. Comienza sus primeras expresiones de duelo y explica el motivo de su llegada a la residencia. La paciente afirma que es consciente de la realidad. "Estoy aquí por una cosa muy gorda". Establece el llanto y la enfermera le ayuda a expresar sus sentimientos. Se ayuda con fotografías y le explica que vive triste. "Lo peor de todo ha sido no poder despedirme de él". Caminan por la residencia y suben y bajan las escaleras con ayuda de la barandilla.

Se pactan los días y horarios de las actividades a realizar en el plan y la enfermera los registra para evitar el olvido de la paciente. 
La enfermera llega a la Residen-

$2^{\circ}$ intervención: (7 de Abril de 2016)

Después de unos días sin verse la paciente espera la llegada de la enfermera.

Se saludan cordialmente con dos besos, salen por la calle más cercana a la Residencia y caminan a ritmo moderado durante aproximadamente 15 minutos. La paciente se apoya en el brazo de la enfermera, refiere sentirse "más segura así".

Entran a una tienda para hacer unas compras y vuelven a la Residencia dando un paseo durante otros 20 minutos.

La paciente se muestra contenta con la compañía y el paseo de la mañana.

Suben a la habitación por las escaleras y ayudada de las barandillas y la paciente vuelve a mostrar las fotos de su hijo, esta vez sin llanto.

Expresa sentimientos de cariño hacia su hijo y su nuera. "Ella siempre le cuidó, es una buena chica". Se despiden hasta el próximo día.

$3^{\mathrm{a}}$ intervención: (14 de Abril de 2016) cia a la hora prevista y la paciente ya está lista para salir a la calle.

Le pide ir a mirar ropa de verano y la enfermera acepta. Caminan durante 20 minutos hasta llegar a la zona de tiendas. "Ahora que llega el verano voy a empezar a ponerme ropa con más color, quizá así me sienta mejor". La paciente relaciona su modo de vestir con sus sentimientos de tristeza, pero va a intentar dar un poco más de luz a su imagen con la llegada del verano. La enfermera le anima a que así sea.

Después de mirar vestidos vuelven conversando a la Residencia aunque la paciente siempre tiene a su hijo presente. No obstante, refiere que ahora está empezando a comer más porque empieza a notar algo de mejoría en su estado de ánimo.

Al llegar, se despiden hasta el próximo día.

$4^{\mathrm{a}}$ intervención: (23 de Abril de 2016)

Como todos los días cuando la enfermera llega a la Residencia, la paciente está preparada para salir.

Caminan durante 20 minutos hasta llegar a una cafetería muy frecuentada por la paciente. "Vengo mucho con mi nuera". Hablan sobre el pasado de la paciente, su juventud y fechas señaladas 
como la de su boda. "Yo siempre he sido muy feliz, pero ahora me está tocando pasarlo mal". La enfermera le anima a que exprese todos sus sentimientos, tanto los positivos como los negativos, ya que la paciente siente una sensación agridulce.

Después de desayunar vuelven a la Residencia caminando durante otros 20 minutos a paso moderado. Una vez allí la paciente agradece a la enfermera las actividades realizadas, refiere sentirse cada día un poco mejor.

Se despiden hasta el próximo día, el último del plan de cuidados.

$5^{\text {a }}$ intervención: (28 de Abril de 2016)

La enfermera llega a la hora de siempre a la Residencia y la paciente le espera preparada. Caminan 15 minutos a ritmo moderado hasta llegar al domicilio de la paciente. "Suelo venir a revisar el buzón". La enfermera le anima a no dejar su hogar olvidado para seguir su vida lo más similar posible a como era antes. Vuelven a conversar acerca del fallecimiento de su hijo mientras caminan durante aproximadamente 10 minutos.

Miran algún escaparate y vuelven a la Residencia por otro camino al habitual. Se despiden mientras la paciente le pide a la enfermera mantener el contac- to. "Muchas gracias por todo, me has ayudado mucho." Se despiden con dos besos.

\section{Actividades del paciente reali-} zadas de manera independiente:

La paciente ha salido a caminar 4-5 veces a la semana durante el plan de movilidad física. Refiere que la mayoría de veces prefiere ir sin la compañía de otros residentes para poder llevar su propio ritmo.

\section{EVALUACIÓN}

Se ha realizado una encuesta para valorar el grado de satisfacción de la paciente respecto al plan de movilidad física explicada a continuación: 


\begin{tabular}{|c|c|c|c|c|c|}
\hline \multirow{2}{*}{ ACTIVIDAD } & \multicolumn{5}{|c|}{ GRADO DE SATISFACCIÓN } \\
\hline & Nada & Poca & Moderada & Bastante & Mucha \\
\hline Idea de realizar el plan & & & & $\mathrm{x}$ & \\
\hline Duración del plan (mes de abril) & & $x$ & & & \\
\hline Efectividad del plan & & & & $\mathrm{X}$ & \\
\hline Días por semana del plan & & $x$ & & & \\
\hline Persona que realiza el plan & & & & & $\mathrm{X}$ \\
\hline Puntualidad & & & & & $\mathrm{x}$ \\
\hline $\begin{array}{l}\text { Lugares donde se han realizado las } \\
\text { actividades }\end{array}$ & & & & $\mathrm{X}$ & \\
\hline Trato enfermera-paciente & & & & & $\mathrm{x}$ \\
\hline ¿Cómo se siente ahora respecto a antes? & & & $\mathrm{X}$ & & \\
\hline ¿Está satisfecho de haber hecho el plan? & & & & & $\mathrm{X}$ \\
\hline ¿Ha notado mejoría? & & & & $x$ & \\
\hline
\end{tabular}

Además se ha vuelto a valorar el estado de la paciente a través de la escala de depresión Yesavage cuya puntuación es de diez, significando depresión establecida, dato no significativo por haber aumentado un solo punto. En el Mini Examen Cognoscitivo de Lobo obtiene una puntuación de treinta y dos, sin deterioro cognitivo.

Tanto las escalas como la encuesta las ha realizado la institución en la que se encuentra la paciente para interferir lo menos posible en las respuestas.

A la vista de los resultados se puede concluir que la paciente ha mejorado su estado de ánimo subjetivo. Por ello está en proceso de lograr el resulta- do esperado. No obstante, valora el tiempo de intervención algo corto en cuanto a duración del plan se refiere por lo que se podría mantener en el futuro.

El grado de satisfacción personal al realizar este plan de cuidados es alto al haber cumplido los objetivos propuestos. Además, es un plan en el que el afecto juega un papel importante, estableciéndose así una buena relación mutua.

\section{Discusión}

La depresión se encuadra dentro de las enfermedades mentales más comunes en las que el paciente pasa por un proceso muchas veces difícil de aceptar. Se encuadran multitud de síntomas 
desempeñando el diagnóstico precoz un

papel muy importante.

Existen escalas que permiten al profesional sanitario valorar y objetivar la depresión y la evolución del paciente a través de la enfermedad.

Se debe realizar un abordaje global a los pacientes mayores con depresión para que las intervenciones de enfermería sean eficaces. No solo hay que potenciar la movilidad, sino también la confianza del paciente en el profesional de enfermería, la expresión de sentimientos y la integración social.

El tratamiento de la depresión encuadra además de psicofármacos otras terapias como planes de cuidados individualizados.

La realización de este plan de cuidados de enfermería ha sido efectiva al no haber requerido gastos materiales extra para su ejecución. No obstante, podría ser continuado por un equipo de Atención Primaria.

La mejora de la movilidad física es un factor positivo que puede ayudar a disminuir la depresión en el paciente geriátrico. 


\section{BiBLIOGRAFÍA}

1. Lobo A. Manual de psiquiatría genera. Madrid: Panamericana; 2012.

2. Ayuso J.L, Ballus C, Barcia D, Cervera S, Conde $\mathrm{V}$, Coullant $\mathrm{J}$ et all. La depresión, un problema crucial en nuestro tiempo. Madrid: Espasa; 1992.

3. Rosenthal L, Carroll-Scott A, Earnshaw V, Santilli A, Ickovics J. The importance of full-time work for urban adults' mental and physical health. Soc Sci Med. 2012 Nov; 75(9): 1692-6.

4. John F. Tratamiento de la depresión recidivante. Barcelona: Ars Medica; 2002.

5. Herrera J. Depresión en el anciano. Aten Primaria. 2000; 26(5): 339-346.

6. Riquelme A, Buendía J, López A. Desarrollo y validación de un instrumento para la evaluación de la depresión en ancianos. Psicothema. 2006; 18(2): 288-292.

7. American Psychiatric Association. Guía clínica para el tratamiento del trastorno depresivo mayor: Barcelona; 2001.

8. Murillo A, Loo I. Influencia de la práctica del ejercicio en la funcionalidad física y mental del adulto mayor. Rev Enferm Inst Mex Seguro Soc. 2007; 15 (1): 11-20

9. René Barrios R, Rolando I, Cardoso L. Beneficios percibidos por adultos mayores incorporados al ejercicio. Rev Cubana Med Gen Integr. 2003; 19(2).

10.Márquez E, Soriano S, García A, Falcon M.P. Depresión en el adulto mayor: frecuencia y factores de riesgo asociados Unidad de Medicina Familiar.

11.Atkins J, Naismith SL, Luscombe GM, Hickie IB. Psychological distress and quality of life in older persons: relative contributions of fixed and modifiable risk factors. BMC Psychiatry. 2013 Oct 8; 13: 249.

12.Siennicki-LAntz A, André-Petersson, L. Wollmer Elmstahl S. Depressive symptoms, atherosclerotic burden and cerebral blood flow disturbances in a cohort of octogenarian men from a general population. BMC Psychiatry. 2013; 13: 347.

13. Litwin H. Physical activity, social network type and depressive symptoms in late life: An analysis of data from the National Social Life, Health and Aging Project. Aging Ment Health. 2012; 16(5): 608-16.

14.Peltzer K, Phaswana-Mafuya N. Depression and associated factors in older adults in South Africa.Glob Health Action. 2013; 6:18871.

15.KArel G. ¿Qué capacidad tienen los enfermeros y los auxiliares de enfermería para reconocer y tratar la depresión en las personas mayores? Informaciones Psiquiátricas. 2009; 195-196.
16. Barriopedro MI, Eraña I, Mallol L. Relación de la actividad física con la depresión y satisfacción con la vida en la tercera edad. Revista de Psicología del Deporte. 2001. 10(2); 239-246.

17. Hernandez R, Prohaska TR, Wang PC, Sarkisian CA. The Longitudinal Relationship Between Depression and Walking Behavior in Older Latinos: The "ICaminemos!" Study. J Aging Health. 2013 Mar; 25(2): 319-41

18.Casas Herrero A, Izquierdo M. Ejercicio físico como intervención eficaz en el anciano frágil. Anales Sis San Navarra. 2012; 35(1): 69-85.

19.Ricardo J, Castells P. Trastornos del equilibrio en el anciano déficit multisensorial. Recomendaciones. 2014.

20.Álvarez A, López S, Hernández J, Sánchez P. La influencia del ejercicio y la actividad física en la salud mental y el bienestar.

21. Mikel Izquierdo M, Cadore E, Casas A. Ejercicio físico en el anciano frágil: una manera eficaz de prevenir la dependencia. Kronos. 2014; 13(1).

22.Dimas C, García H. Intervenciones efectivas en adultos mayores con depresión. Rev Paraninfo Digital. 2011; 12.

23. Correia J, Ravasco P. Weight changes in Portuguese patients with depression: which factors are involved? Nutr J. 2014 Dec 16; 13(1): 117.

24.Gale CR, Allerhand M, Sayer AA, Cooper C, Deary IJ. The dynamic relationship between cognitive function and walking speed: the English Longitudinal Study of Ageing. Age (Dordr). 2014; 36(4): 9682.

25.Rosenberg D, Kerr J, James F. Gregory J, Karen Calfas, Patrick K. Promoting Walking Among Older Adults Living in Retirement Communitie. Journal of Aging and Physical Activity. 2012; 20: 379-394.

26. Maldonado G, Carbajal F, Rivera P, Castro R. Beneficios que percibe el adulto mayor al integrarse a un grupo de ayuda dirigido por personal de enfermería. Rev Enferm IMSS Méx. 2015; 23(1): 31-36.

27.Jürschik $P$, Botigué $T$, Nuin $C$, Lavedán $A$. Estado de ánimo caracterizado por soledad y tristeza factores relacionados en personas mayores. Gerokomos. 2013; 24(1): 14-17.

28. Silva J, Castro J, Coelho S, Fernandes A, Partezani R. Factores asociados a la capacidad funcional en adultos mayores atendidos en un hospital de día de geriatría. Nure Inv. 2015; 12(78): 1-9.

29.Martínez de la Iglesia J, Onís Vilches MC, Dueñas Herrero R, Aguado Taberna C, Albert Colomer C, Luque Luque R. Versión española del cuestionario de Yesevage abreviado (GDS) para el cribado de depresión en mayores de 65 años: Adaptación y validación. Medifam. 2002; 12: 620-30.

30.López J, Martí A, Instituto de Medicina Legal de Cataluña, Mini-examen cognoscitivo (MEC) artícu- 
lo en Revista Española de Medicina Legal. 2011; 37(3):122-127.

31. Herdman T, Kamitsuru S. NANDA international. Diagnósticos enfermeros. Definiciones y clasificación. Barcelona: Elsevier; 2018-2020.

32.Butcher HK, Bulechek GM, Dochterman JM, Wagner CM. Clasificación de Intervenciones de Enfermería (NIC). Madrid: Elsevier; $7^{a}$ ed. 2018.
33.Moorhead S, Swanson E, Johnson M, Maas ML. Clasificación de Resultados de Enfermería (NOC). Madrid: Elsevier; $6^{a}$ ed. 2018.

\section{TABLAS Y ANEXOS}

ANEXO 1

\begin{tabular}{|l|l|}
\hline Humor & $\begin{array}{l}\text { Durante la mayor parte de los días el paciente notifica un estado } \\
\text { de ánimo depresivo. }\end{array}$ \\
\hline Intereses & $\begin{array}{l}\text { Durante la mayor parte de los días el interés o el placer se ven } \\
\text { notablemente reducidos. }\end{array}$ \\
\hline Alimentación y peso & $\begin{array}{l}\text { Aunque no se siga una dieta, el paciente experimenta una } \\
\text { pérdida o un aumento de peso significativo. }\end{array}$ \\
\hline Sueño & $\begin{array}{l}\text { Casi todos los días el paciente duerme demasiado o no duerme } \\
\text { lo suficiente. }\end{array}$ \\
\hline Actividad motora & $\begin{array}{l}\text { Casi todos los días, se observa que el paciente se mueve de } \\
\text { forma agitada o lenta. }\end{array}$ \\
\hline Fatiga & Casi todos los días el paciente se cansa o pierde energía. \\
\hline Autoestima & $\begin{array}{l}\text { Casi todos los días el paciente no se valora o se siente culpable } \\
\text { sin motivo. }\end{array}$ \\
\hline Concentración & $\begin{array}{l}\text { Casi todos los días el paciente se siente indeciso o se le hace } \\
\text { difícil pensar o concentrarse. }\end{array}$ \\
\hline Euerte & $\begin{array}{l}\text { El paciente tiene pensamientos recurrentes sobre la muerte o } \\
\text { suicion }\end{array}$ \\
\hline
\end{tabular}

Los nueve síntomas posibles de episodio depresivo según los criterios DSM-IV. 
ANEXO 2

\begin{tabular}{|c|c|c|}
\hline PREGUNTA & SI & NO \\
\hline $\begin{array}{l}\text { ¿Está satisfecho son su } \\
\text { vida? }\end{array}$ & 0 & 1 \\
\hline $\begin{array}{l}\text { ¿Ha renunciado a } \\
\text { muchas actividades? }\end{array}$ & 1 & 0 \\
\hline $\begin{array}{l}\text { ¿Siente que su vida } \\
\text { está vacía? }\end{array}$ & 1 & 0 \\
\hline $\begin{array}{l}\text { ¿se encuentra a } \\
\text { menudo aburrido/a? }\end{array}$ & 1 & 0 \\
\hline $\begin{array}{l}\text { ¿Tiene a menudo buen } \\
\text { ánimo? }\end{array}$ & 0 & 1 \\
\hline $\begin{array}{l}\text { ¿Teme que algo malo le } \\
\text { pase? }\end{array}$ & 1 & 0 \\
\hline $\begin{array}{l}\text { ¿Se siente feliz muchas } \\
\text { veces? }\end{array}$ & 0 & 1 \\
\hline $\begin{array}{l}\text { ¿Se siente a menudo } \\
\text { abandonado? }\end{array}$ & 1 & 0 \\
\hline $\begin{array}{l}\text { ¿Prefiere quedarse en } \\
\text { casa a salir? }\end{array}$ & 1 & 0 \\
\hline $\begin{array}{l}\text { ¿Cree tener más } \\
\text { problemas dememoria } \\
\text { que la mayoría de la } \\
\text { gente? }\end{array}$ & 1 & 0 \\
\hline $\begin{array}{l}\text { ¿Piensa que es } \\
\text { maravilloso vivir? }\end{array}$ & 0 & 1 \\
\hline $\begin{array}{l}\text { ¿Le cuesta iniciar } \\
\text { nuevos proyectos? }\end{array}$ & 1 & 0 \\
\hline $\begin{array}{l}\text { ¿Se siente lleno/a de } \\
\text { energía? }\end{array}$ & 0 & 1 \\
\hline $\begin{array}{lll}\text { ¿Siente } & \text { sue } \\
\text { situación } & \text { su } \\
\text { desesperanzada? } & \text { es } \\
\end{array}$ & 1 & 0 \\
\hline $\begin{array}{l}\text { ¿Cree que mucha gente } \\
\text { está mejor que usted? }\end{array}$ & 1 & 0 \\
\hline
\end{tabular}

Test de Yesavage de la paciente con una puntuación total de 9 puntos, lo que indica depresión leve. 